\title{
INTEGRAL observation of 3EG J1736-2908 ^
}

\author{
G. Di Cocco ${ }^{1}$, L. Foschini ${ }^{1}$, P. Grandi ${ }^{1}$, G. Malaguti ${ }^{1}$, A. J. Castro-Tirado ${ }^{2}$, S. Chaty ${ }^{3,4}$, A. J. Dean ${ }^{5}$, N. Gehrels ${ }^{6}$, \\ I. Grenier ${ }^{3,4}$, W. Hermsen ${ }^{7}$, L. Kuiper ${ }^{7}$, N. Lund ${ }^{8}$, and F. Mirabel ${ }^{3}$ \\ ${ }^{1}$ Istituto di Astrofisica Spaziale e Fisica Cosmica (IASF) del CNR - Sezione di Bologna, via Gobetti 101, \\ 40129 Bologna, Italy \\ e-mail: dicocco@bo.iasf.cnr.it \\ 2 Instituto de Astrofísica de Andalucía (IAA-CSIC), PO Box 03004, 18080 Granada, Spain \\ 3 CEA Saclay, DSM/DAPNIA/SAp (CNRS FRE 2591) 91191 Gif-sur-Yvette Cedex, France \\ ${ }^{4}$ Université Paris 7 Denis-Diderot 2 place Jussieu 75251 Paris Cedex 05, France \\ 5 Department of Physics and Astronomy, University of Southampton, Southampton SO17 1BJ, UK \\ ${ }^{6}$ NASA Goddard Space Flight Center, Laboratory for High Energy Astrophysics, Code 661, Greenbelt, MD, USA \\ 7 SRON National Institute for Space Research, Sorbonnelaan 2, 3584 CA Utrecht, The Netherlands \\ ${ }^{8}$ Danish Space Research Institute, Juliane Maries Vej 30, 2100 Copenhagen, Denmark
}

Received 19 March 2004 / Accepted 4 June 2004

\begin{abstract}
The possible identification by INTEGRAL of the EGRET source 3EG J1736-2908 with the active galactic nucleus GRS 1734-292 is discussed. The latter was discovered in 1990 and later identified with a Seyfert 1 galaxy. At the time of the compilation of the 3rd EGRET Catalog, it was not considered as a possible counterpart of the source 3EG J1736-2908, which remained unidentified. A detailed multiwavelength study of the EGRET error circle is presented, by including archival radio, soft- and hard-X observations, suggesting that GRS1734-292 could be a likely counterpart of 3EG J1736-2908, even though this poses very interesting questions about the production mechanisms of $\gamma$-rays with energies greater than $100 \mathrm{MeV}$.
\end{abstract}

Key words. gamma-rays: observations - X-rays: galaxies - galaxies: active - galaxies: individual: GRS1734-292

\section{Introduction}

The Third Energetic Gamma-Ray Experiment Telescope (EGRET) Catalogue of high-energy $(E>100 \mathrm{MeV}) \gamma$-ray sources (Hartman et al. 1999) refers to the observations carried out by the EGRET experiment on-board the Compton Gamma-Ray Observatory (CGRO) between April 1991 and October 1995. It contains 271 point sources, including: 1 solar flare, the Large Magellanic Cloud, 5 pulsars, the radio galaxy Centaurus A, and 66 high-confidence identifications of blazars. In addition, 27 lower-confidence blazar identifications are included in the catalog, which leaves 170 sources unidentified. Since the publication of the catalog, a lot of effort has been dedicated to the identification of the 170 unidentified EGRET sources (e.g. Grenier 2003). While most of the extragalactic counterparts are blazars, two possible identifications of EGRET sources with radiogalaxies have been recently proposed (Combi et al. 2003; Mukherjee et al. 2003), in addition to Centaurus A. However, the multiwavelength counterpart

^ Based on observations with INTEGRAL, an ESA project with instruments and science data center funded by ESA member states (especially the PI countries: Denmark, France, Germany, Italy, Switzerland, Spain), Czech Republic and Poland, and with the participation of Russia and the USA. search is hampered by the limited EGRET point spread function (PSF), which gives error radii of $0.5^{\circ}-1^{\circ}$.

The INTEGRAL satellite (Jensen et al. 2003) was launched on October 17th, 2002. During the first year of scientific operations, more than half of the INTEGRAL Core Programme (CP, Winkler et al. 2003) was dedicated to a deep exposure of the Galactic central radian, and to regular weekly scans of the Galactic plane.

The IBIS imager (Ubertini et al. 2003), one of the two INTEGRAL main telescopes, combines for the first time broad-band high-energy coverage $(15 \mathrm{keV}-10 \mathrm{MeV})$ with imaging capability. IBIS has a large field of view $\left(19^{\circ} \times 19^{\circ}\right.$ at half response), and a fine angular resolution of $12^{\prime}$, sampled in $5^{\prime}$ pixels in the low energy $(0.015-1 \mathrm{MeV})$ layer ISGRI (Lebrun et al. 2003), and in $10^{\prime}$ pixels in the highenergy (0.175-10 MeV) detector PICsIT (Di Cocco et al. 2003). ISGRI reaches a point source location accuracy better than $1^{\prime}$ for a $\sim 30 \sigma$ detection (Gros et al. 2003). These unprecedented characteristics for a gamma-ray telescope clearly make IBIS one of the best instruments available to date to search for counterparts of unidentified EGRET sources.

Here we present the first results of a program set up to search for the identification of the unidentified EGRET sources located in the Galactic plane. The first INTEGRAL 
proposed identification of an unidentified EGRET source concerns 3EG J1736-2908 and the active galactic nucleus GRS1734-292. 3EG J1736-2908 was originally detected by EGRET with a $\sim 6 \sigma$ with a $95 \%$ confidence circle of $\theta_{95}=$ $0.62^{\circ}$ in radius, at a position very close to the Galactic Centre (Hartman et al. 1999). The spectrum showed a photon in$\operatorname{dex} \Gamma=2.18 \pm 0.12$ and a flux for $E>100 \mathrm{MeV}$ of $(3.3 \pm 0.6) \times 10^{-7}$ photons $\mathrm{cm}^{-2} \mathrm{~s}^{-1}$, averaged over the whole viewing period.

EGRET observed this source in 23 separate viewing periods (Hartman et al. 1999). The measured fractional variability index, defined as $\delta=\sigma / \mu$ (Nolan et al. 2003), where $\sigma$ and $\mu$ are the flux standard deviation and average values respectively, is $\delta=0.64_{-0.33}^{+0.48}$. This value is larger than the fractional variability expected for steady sources, $\delta<0.14$, thus indicating that 3EG J1736-2908 is variable. A similar conclusion was independently obtained by McLaughlin et al. (1996) using a different method to quantify the EGRET flux variability.

It is worth noting that the two established source classes able to generate high-energy $\gamma$-rays in the EGRET range, pulsars and active galactic nuclei (AGN), are known to produce steady and strongly variable fluxes, respectively.

\section{INTEGRAL/IBIS observations and data analysis results}

In order to allow imaging with the SPI instrument (Vedrenne et al. 2003), the INTEGRAL observing strategy consists of several off-target pointings of the spacecraft axis following a dithering pattern with steps of $2^{\circ}$ (Jensen et al. 2003). The $\mathrm{CP}$ Galactic Plane scans are performed by INTEGRAL following a sawtooth pattern with $21^{\circ}$ of inclination with respect to the Galactic plane and with a shift of $27.5^{\circ}$ in Galactic longitude between two scans (Winkler et al. 2003). Each scan consists of an alternated sequence of very short $(\approx 120$ s) slews and staring pointings of $2200 \mathrm{~s}$ (each staring pointing is called Science Window, $\mathrm{ScW}$ ). Sometimes it occurs that in the consolidation process at the INTEGRAL Science Data Center (ISDC, Courvoisier et al. 2003), two nearby ScWs are merged, resulting in a longer $\mathrm{ScW}(\approx 5000 \mathrm{~s})$.

The single CP ScWs are sequentially analysed, and all the detected sources are cross-correlated with the 3rd EGRET Catalogue to look for source identifications. All the INTEGRAL data analysis described in the present work has been done with the most recent version of the Offline Standard Analysis $\left(\mathrm{OSA}^{1}\right)$ v. 3.0 (Goldwurm et al. 2003a). This strategy is useful to search for sources in outburst.

A source inside the error contours of the EGRET unidentified source 3EG J1736-2908 was found in ScW 57 of revolution 61, with a flux of $(2.1 \pm 0.3) \times 10^{-10} \mathrm{erg} \mathrm{cm}^{-2} \mathrm{~s}^{-1}$ (or $28 \pm 4 \mathrm{mCrab}^{2}$ ) in the $20-40 \mathrm{keV}$ energy band and signal-tonoise ratio of about $10 \sigma$. To take into account the uncertainties

\footnotetext{
1 Available at

http://isdc.unige.ch/index.cgi?Soft+download

$21 \mathrm{mCrab}_{20-40 \mathrm{keV}}=7.6 \times 10^{-12} \mathrm{erg} \mathrm{cm}^{-2} \mathrm{~s}^{-1} ; 1 \mathrm{mCrab}_{40-60 \mathrm{keV}}=$ $4.2 \times 10^{-12} \mathrm{erg} \mathrm{cm}^{-2} \mathrm{~s}^{-1}$, by assuming $F_{\mathrm{Crab}}(E)=9.6$. $E^{-2.1} \mathrm{ph} \mathrm{cm}^{-2} \mathrm{~s}^{-1} \mathrm{keV}^{-1}$.
}

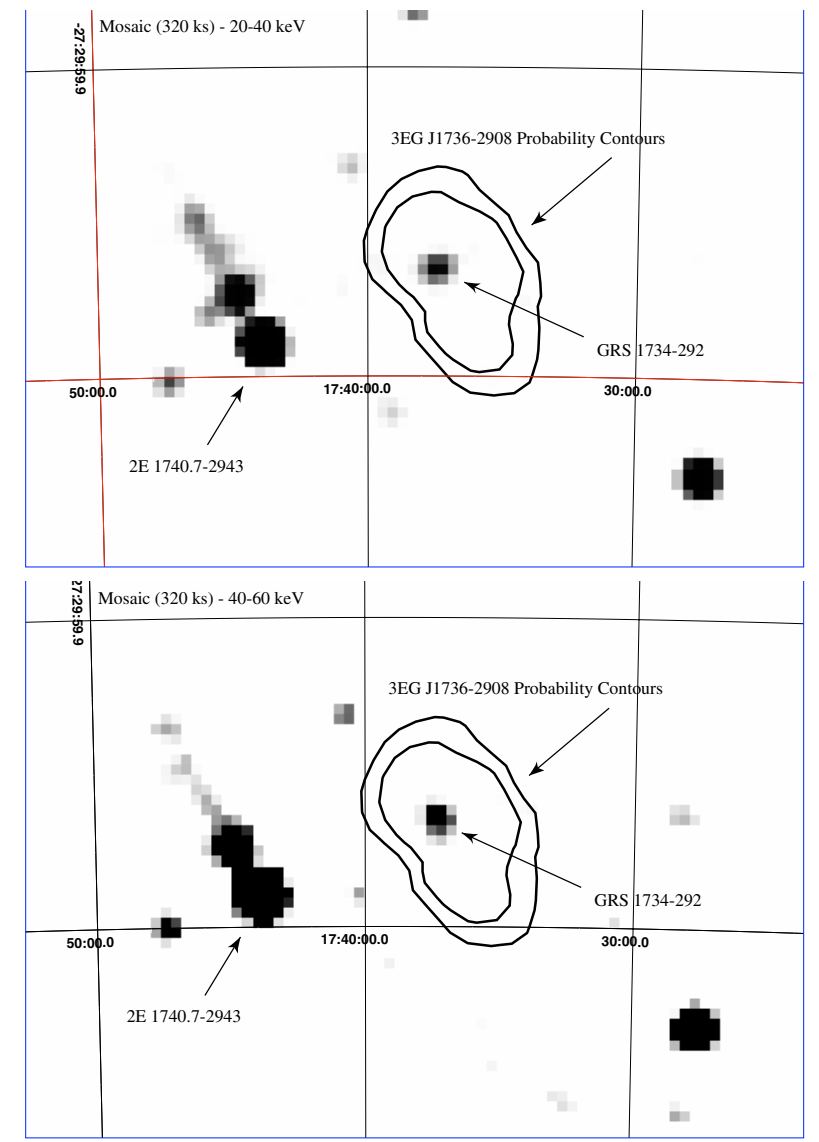

Fig. 1. IBIS/ISGRI deconvolved images of the 3EG J1736-2908 sky region: mosaic of the region with exposure of $320 \mathrm{ks}$, in the energy bands 20-40 (left) and 40-60 (right) keV. The 3EG J1736-2908 probability contours at $95 \%$ and $99 \%$ confidence level are shown. The detected source position inside the probability contours is coincident with the GRANAT source GRS 1734-292 (see text)

in the count rate estimation of the present version of OSA for ISGRI (see Goldwurm et al. 2003b), we adopted the procedure outlined by Goldoni et al. (2003) for the analysis of the off-axis sources. The average fluxes in mCrab have been obtained using calibration observations of the Crab Nebula at similar off-axis angles and adding a 5\% systematic error.

To strengthen the detection significance and to search for other possible counterparts within the EGRET error circle, all the ScWs containing the EGRET region within $10^{\circ}$ from the centre of the field of view (FOV) were selected and summed together. The EGRET error circle of 3EG J1736-2908 was observed by INTEGRAL between revolution 53 and 63, in March-April 2003, for a global exposure of $\approx 320 \mathrm{ks}$. The resulting images are shown in the upper $(20-40 \mathrm{keV})$ and lower $(40-60 \mathrm{keV})$ panels of Fig. 1. The source position ( $90 \%$ confidence radius $\left.=1.2^{\prime}\right)$ obtained with this longer exposure, $\alpha=17: 37: 27$ and $\delta=-29: 08: 24$ (J2000), is fully compatible with the single $\mathrm{ScW}$ detection, while the detection significances are now $17 \sigma$, and $8.5 \sigma$, in the $20-40$ and $40-60 \mathrm{keV}$ bands, respectively, and the associated flux $(4.9 \pm$ $0.4) \times 10^{-11} \mathrm{erg} \mathrm{cm}^{-2} \mathrm{~s}^{-1}$ and $(2.1 \pm 0.3) \times 10^{-11} \mathrm{erg} \mathrm{cm}^{-2} \mathrm{~s}^{-1}$. 
The observed differences in the derived flux values could be an indication for strong flux variability. The source was only detected in ScW 57, while in all the other ScW it was below the detection threshold, with a flux upper limit $(3 \sigma)$ for a single typical ScW of about $8 \times 10^{-11} \mathrm{erg} \mathrm{cm}^{-2} \mathrm{~s}^{-1}$.

In this scenario, the positive detection in ScW 57 (elapsed time $\simeq 7000 \mathrm{~s}$, effective exposure $5000 \mathrm{~s}$ ) has then to be associated with the source being in outburst, while in the deep exposure $(\sim 320 \mathrm{ks})$ the source becomes visible also in its quiescent state. The robustness of this result has been confirmed by crosschecking the fractional flux variations of the three strongest sources in the field. One of these source was steady, while the other two, although not constant, showed variability patterns different from the EGRET candidate counterpart.

The centroid position of the IBIS source is $<0.6^{\prime}$ from the known GRANAT source GRS 1734-292, well within the IBIS point source location accuracy for the observed source strength $\left(\simeq 2^{\prime}-3^{\prime}\right)$. This therefore gives a first, strong, indication that GRS 1734-292 is the source detected by INTEGRAL.

The same source is also clearly visible in the mosaics of the Galactic Centre region presented by Paizis et al. (2003), in the field of view of the black hole candidate H1743-322 (Parmar et al. 2003), and in the IBIS/ISGRI survey of the Galactic Centre region (Revnivtsev et al. 2004). It is worth noting that in the $2 \mathrm{Ms}$ IBIS/ISGRI mosaic by Revnivtsev et al. (2004) the source is detected in the $18-60 \mathrm{keV}$ energy band at $35 \sigma$ level with a flux of $\sim 7 \times 10^{-11} \mathrm{erg} \mathrm{cm}^{-2} \mathrm{~s}^{-1}$, consistent with the present results. Moreover, it is easy to recognize that in the $2 \mathrm{Ms}$ IBIS/ISGRI survey there are no other sources in the EGRET error circle, down to a flux limit of $\simeq 1.3 \times 10^{-11} \mathrm{erg} \mathrm{cm}^{-2} \mathrm{~s}^{-1}(6.5 \sigma)$.

Finally, the data from the INTEGRAL X-ray instrument JEM-X (energy range: $3-35 \mathrm{keV}$; Lund et al. 2003), show the presence of only one source $(\approx 10 \mathrm{mCrab}$ above $8 \mathrm{keV})$ within the $95 \%$ confidence contours of 3EG J1736-2908, with a mosaic having effectively a $20 \mathrm{ks}$ exposure time. The position of this source is compatible with GRS1734-292 within the angular resolution of JEM-X at this flux level $\left(\approx 2^{\prime}\right)$.

\section{GRS 1734-292}

GRS 1734-292 was originally discovered by the codedmask telescope ART-P (Sunyaev et al. 1990) onboard the GRANAT satellite (Sunyaev 1990). Early analysis in the 3-30 keV energy band showed a power law spectrum with $\Gamma=2.0 \pm 0.1$, an absorbing column of $\simeq 6 \times 10^{22} \mathrm{~cm}^{-2}$, and the flux at $10 \mathrm{keV}$ was $(3.2 \pm 0.6) \times 10^{-4}$ photons $\mathrm{cm}^{-2} \mathrm{~s}^{-1}$ (Pavlinsky et al. 1994), which corresponds to a $20-40 \mathrm{keV}$ flux of $\sim 4 \times 10^{-11} \mathrm{erg} \mathrm{cm}^{-2} \mathrm{~s}^{-1}$. In 1992, GRS 1734-292 experienced an outburst in the 40-400 keV energy band, during which a flux variation by a factor $\sim 2$ in a few days was detected with the coded-mask telescope SIGMA (Paul et al. 1991) onboard the GRANAT satellite (Churazov et al. 1992).

Pavlinsky et al. (1994) estimated the $90 \%$ confidence level error radius of GRS 1734-292 as 95'. This value was later reduced to $3^{\prime \prime}$ by the ROSAT observation of Barret \& Grindlay (1996). The arcsecond level position allowed to find the optical counterpart. GRS 1734-292 was optically classified by
Martí et al. (1998) as a Seyfert 1, with $m_{\mathrm{V}}=21.0 \pm 0.3$, on the basis of the strong and very broad emission of blended $\mathrm{H} \alpha$ and $\mathrm{N}[\mathrm{II}]$ lines (the FWZI of the blended line is $\sim 2 \times 10^{4} \mathrm{~km} \mathrm{~s}^{-1}$ ). The redshift was measured as $z=0.0214$.

In a VLA observation, GRS 1734-292 was resolved with a clear bipolar jet-like structure (Martí et al. 1998). Its radio spectrum could be well described by a power law $S_{v} \propto v^{-\alpha}$ with $\alpha=0.75 \pm 0.03$. The total (core plus jets) flux densities at 1.4 and $5 \mathrm{GHz}$ are $\simeq 59$ and $\simeq 23 \mathrm{mJy}$, respectively.

Sakano et al. (2002) reported the detection of GRS 1734-292 with ASCA. The source was observed twice, in 1998 and 1999, with a $0.7-10 \mathrm{keV}$ flux of 4.3 and $3.1 \times 10^{-11} \mathrm{erg} \mathrm{cm}^{-2} \mathrm{~s}^{-1}$, respectively. The spectrum was well fitted by a power law with slope $\Gamma \sim 1.4 \div 1.5$, absorbed by a column density $N_{\mathrm{H}}<2.1 \times 10^{22} \mathrm{~cm}^{-2}$. The value of $N_{\mathrm{H}}$ was well constrained by ASCA only in one occasion giving a value of $1.7_{-0.3}^{+0.4} \times 10^{22} \mathrm{~cm}^{-2}$. This is above the galactic hydrogen column density of $\simeq 0.8 \times 10^{22} \mathrm{~cm}^{-2}$ in the direction of the source measured by Dickey \& Lockman (1990), but consistent with the measurement of the absorption column of $1.4 \times 10^{22} \mathrm{~cm}^{-2}$ by Schlegel et al. (1998), based on COBE and IRAS data. Indeed, given the position of the source very close to the Galactic centre, the measurements of the absorption column have to be taken with great caution. For example, independent measurements performed in the optical band with three different methods (Martí et al. 1998) gave $N_{\mathrm{H}}=(1.0 \pm 0.2) \times 10^{22} \mathrm{~cm}^{-2}$.

\section{X-ray and radio cross-correlations and counterparts}

The error circle of 3EG J1736-2908 contains no obvious galactic EGRET counterparts. The apparent association with the pulsar PSR J1736-2843 is almost certainly due to a chance alignment (Kramer et al. 2003) on the basis of the variability indicator defined by McLaughlin et al. (1996). Moreover, an independent variability indicator (Nolan et al. 2003) also supports the hypothesis for a non pulsar origin for 3EG J1736-2908. On the other hand, Pulsar Wind Nebulae (PWNe) could be responsible of strong emission in the EGRET range with a variability consistent with what detected for 3EG J1736-2908. However none of the PWNe detected in the Galactic Plane falls within the 3EG J1736-2908 error circle (Nolan et al. 2003; Grenier 2003).

Further possibility of galactic counterparts, which are suggested to be pulsar tracers, can be also excluded. No WolfRayet stars, Of stars, SNRs and OB associations are present within the 95\% confidence contours of 3EG J1736-2908 (Romero et al. 1999).

The fractional variability of 3EG J1736-2908 is consistent with typical values expected for quasars and BL Lacs (Nolan et al. 2003). The principal method of identification of highlatitude EGRET sources relies on finding positional coincidences with flat spectrum radio loud AGNs. The NRAO/VLA Sky Survey (NVSS) catalog (Condon et al. 1998) contains, in the error circle of 3EG J1736-2908, 85 radio sources. All the sources are radio-weak, with typical fluxes around 20-50 mJy at $1.4 \mathrm{GHz}$. Even in the assumption of a flat radio spectrum, 
this implies weak $5 \mathrm{GHz}$ flux densities $<200 \mathrm{mJy}$, i.e. below the lowest fluxes measured for identified EGRET blazars (Wallace et al. 2002). Torres et al. (2001) have explored the error circles of all low galactic latitude unidentified EGRET sources, listing all significant point-like radio-sources having flux densities above $30 \mathrm{mJy}$ at least at one wavelength. The only two strong radio emitters they found are: PNESO 359.3+01.4 and $\mathrm{OH} 359.140+01.137$. The first is a planetary nebula, while the latter is a molecular cloud. Both sources can be excluded as responsible for the gamma-ray emission on the basis of the observed EGRET variability (Nolan et al. 2003).

We have also cross-correlated all the ROSAT X-ray satellite catalogs available in the HEASARC archive with the $95 \%$ confidence EGRET error radius of 3EG J1736-2908. This resulted in 16 sources, only one of which has a radio counterpart in the NRAO/VLA Sky Survey (NVSS) catalog (Condon et al. 1998): the GRANAT source GRS 1734-292.

The Galactic Centre region was also scanned by the ASCA X-ray satellite (see Sect. 3, Sakano et al. 2002). We have searched the 3EG J1736-2908 error circle and found five ASCA sources, only two of which have a radio counterpart in the NVSS catalog: again GRS1734-292, and AXJ1734.5-2915. The latter is very weak both in radio and X-rays, with a $1.4 \mathrm{GHz}$ flux density of $3.4 \mathrm{mJy}$, while the ASCA X-ray flux $\left(F_{0.7-10 \mathrm{keV}}=3 \times 10^{-13} \mathrm{erg} \mathrm{cm}^{-2} \mathrm{~s}^{-1}\right)$ is a factor $\simeq 100$ less than what was measured for GRS 1734-292.

We thus conclude that the EGRET error circle does not contain any obvious blazar-like candidate. The only source which shows a broad band spectrum from radio to soft (ROSAT) and hard (ASCA, GRANAT, INTEGRAL) X-rays, is GRS 1734-292.

\section{Discussion}

During its Galactic Plane scans, the IBIS imager and the JEM-X monitor on-board INTEGRAL detected only one source within the 3EG J1736-2908 error circle. In IBIS the source was first detected, between 20 and $40 \mathrm{keV}$ in a snap-shot ( $\sim 5000 \mathrm{~s})$ observation in one ScW only. A longer integration of $320 \mathrm{ks}$ allowed the detection of the source at much higher significance and also in the $40-60 \mathrm{keV}$ band. ScW level monitoring suggests a possible short-time (few hours) variability.

The INTEGRAL results together with the measured EGRET variability (Nolan et al. 2003) seem to indicate an identification of 3EG J1736-2908 with GRS 1734-292. Moreover, the multiwavelength search in the probability contours of 3EG J1736-2908 did not reveal the presence of possible blazarlike radio-loud objects with spectral characteristics similar to the already identified EGRET sources. This scenario would leave GRS 1734-292 as the most likely AGN to be associated with the EGRET source. Sazonov et al. (2004) have recently calculated the probability to find by chance an EGRET source consistent with the position of GRS1734-292 to be only 3\%.

The non-classical blazar nature of GRS 1734-292 is not necessarily a problem. In fact, the radiogalaxy NGC 6251, showing a jet forming a large angle with the line of sight, has been proposed for an association with the EGRET source 3EG J1621+8203 (Mukherjee et al. 2002). However, this object is a strong radio emitter, with a core $1.4 \mathrm{GHz}$ flux density $>800 \mathrm{mJy}$, and its spectral energy distribution is well fitted, from radio to gamma-rays, by a double peak synchrotron-self-Compton (SSC) model typical of jet-dominated AGNs (Guainazzi et al. 2003; Chiaberge et al. 2003). On the other hand, an association of another EGRET source, 3EG J1735-1500 with a radio-faint galaxy has recently been proposed (Combi et al. 2003).

The observed radio faintness of GRS $1734-292, F_{1.4 \mathrm{GHz}} \simeq$ $50 \mathrm{mJy}$, combined with the steepness of the radio spectrum, makes it difficult to explain the EGRET emission in terms of the inverse Compton upscattered component from a jet. In addition, the derived spectral index connecting the radio and optical bands is $\alpha_{\mathrm{RO}}<0.16$ (Sambruna et al. 1996). This value characterizes GRS1734-292 as a radio-quiet source (radio-loud objects have by definition $\alpha_{\mathrm{RO}}>0.2$ ). Note, for example, for NGC 6251, $\alpha_{\mathrm{RO}}=0.7$. However, the $\alpha_{\mathrm{RO}}$ value strongly depends on the assumed line-of-sight optical absorption, and since GRS 1734-292 lies close to the Galactic Centre, the uncertainty in its associated $A_{\mathrm{V}}$ value is high. In fact, in the most conservative scenario, i.e. assuming a value for $A_{\mathrm{V}}$ as low as 4 , corresponding to $N_{\mathrm{H}}=0.8 \times 10^{22} \mathrm{~cm}^{-2}$ (Dickey \& Lockman 1990), the resulting spectral index is $\alpha_{\mathrm{RO}}=0.32$, thus satisfying the condition for radio loudness.

On the other hand, the Spectral Energy Distribution (SED) of GRS 1734-292 shown in Fig. 2 is intriguing. We point out that radio fluxes shown in the SED include the contribution of the whole radio source, i.e. both core and jets. Since the jet emission is mainly of synchrotron nature, the radio slope would be steeper by taking into account only the core of the source, and the radio spectrum would then be more naturally aligned towards the infrared data, pointing towards a synchrotron origin. It appears from the SED that ASCA, ART-P, INTEGRAL and SIGMA spectra are fully compatible. Multi-wavelength data, from optical to gamma-ray, resemble a typical two-peak blazar shape. The first peak will be in the near-infrared/optical domain, however its precise location is hampered by the uncertainty in the column density, as is visible from the two infrared/optical spectra derived with different estimates of column density. The second peak will be between SIGMA and EGRET data. Simultaneous multi-wavelength data would help in constraining the accurate shape of GRS 1734-292's SED.

In conclusion, the INTEGRAL scans of the Galactic Centre regions indicate that GRS 1734-292 is a possible counterpart candidate of the unidentified EGRET source 3EG J1736-2908. GRS 1734-292, optically classified as Seyfert 1 and weak radio emitter, is difficult to reconcile with a picture, in which the radio to $\gamma$-ray emission is completely explained in term of strongly beamed non-thermal radiation. In the case that future deeper and higher angular resolution observations in the EGRET band (GLAST, AGILE) will confirm the present scenario, new possible models for $\gamma$-ray emission from AGN will have to be explored.

Acknowledgements. The italian participation to the INTEGRAL/IBIS project is financed by the Italian Space Agency (ASI). This work has made use of public data obtained from the High Energy Astrophysics Science Archive Research Centre (HEASARC), provided by NASA Goddard Space Flight Centre. 


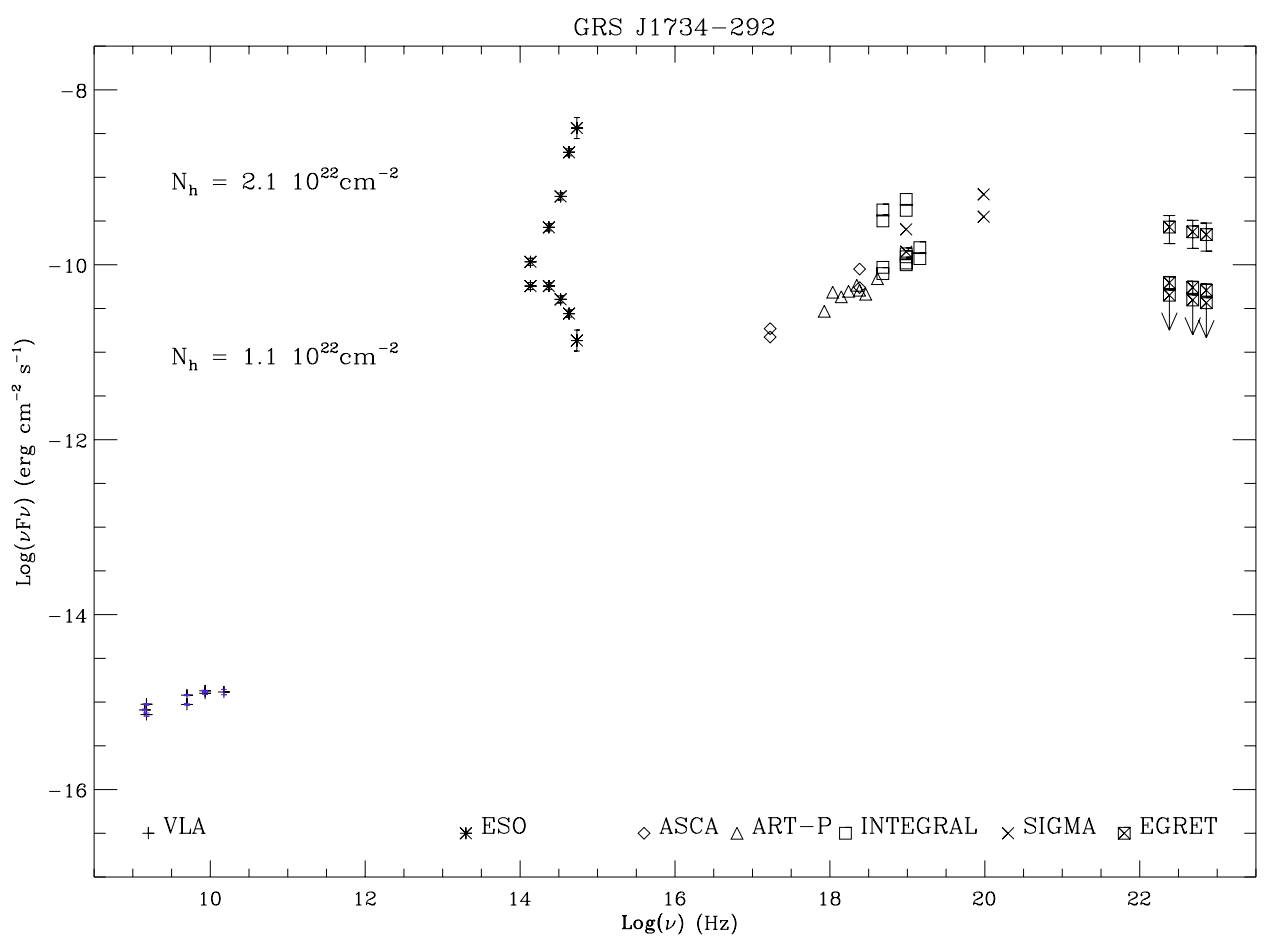

Fig. 2. Spectral Energy Distribution (SED) of GRS 1734-292. We show here all published data: VLA and ESO (Martí et al. 1998), ASCA (Sakano et al. 2002), ART-P (Pavlinsky et al. 1994), INTEGRAL (present paper), SIGMA (Churazov et al. 1992) and EGRET (Hartman et al. 1999). Infrared and optical data from ESO are corrected with two different absorptions, respectively $1.1 \times 10^{22}$ and $2.1 \times 10^{22} \mathrm{~cm}^{-2}$. The data include minimum, mean and maximum observed values, in order to show the interval of variation of GRS 1734-292 from non-detection to flares, at different epochs. If not visible, error bars are smaller than size of symbols.

\section{References}

Barret, D., \& Grindlay, J. E. 1996, A\&A, 311, 239

Cardelli, J. A., Clayton, G. C., \& Mathis, J. S. 1989, ApJ, 345, 245

Chiaberge, M., Gilli, R., Capetti, A., \& Macchetto, F. D. 2003, ApJ, 597,166

Churazov, E., Gilfanov, M., Cordier, B., \& Schmitz-Fryasse, M. C. 1992, IAU Circular, 5623

Combi, J. A., Romero, G. E., Paredes, J. M., Torres, D. F., \& Ribó, M. 2003, ApJ, 588, 731

Condon, J. J., Cotton, W. D., Greisen, E. W., et al. 1998, AJ, 115, 1693

Courvoisier, T. J.-L., Walter, R., Beckmann, V., et al. 2003, A\&A, 411, L53

Dickey, J. M., \& Lockman, F. J. 1990, ARA\&A, 28, 215

Di Cocco, G., Caroli, E., Celesti, E., et al. 2003, A\&A, 411, L189

Goldoni, P., Bonnet-Bidaud, J. M., Falanga, M., \& Goldwurm, A. 2003, A\&A, 411, L399

Goldwurm, A., David, P., Foschini, L., et al. 2003a, A\&A, 411, L223

Goldwurm, A., Gros, A., Goldoni, P., et al. 2003b, IBIS/ISGRI Instrument Specific Software Scientific Validation Report, v. 1.0

Grenier, I. A. 2003, Proc. Texas Symposium 2002, Firenze (Italy) [arXiv: astro-ph/0303498]

Gros, A., Goldwurm A., Cadolle-Bel, M., et al. 2003, A\&A, 411, L179

Guainazzi, M., Grandi, P., Comastri, A., \& Matt, G. 2003, A\&A, 410, 131

Jensen, P. L., Clausen, K., Cassi, C., et al. 2003, A\&A, 411, L7

Kramer, M., Bell, J. J., Manchester, R. N., et al. 2003, MNRAS, 342, 1299

Hartman, R. C., Bertsch, D. L., Bloom, S. D., et al. 1999, ApJS, 123, 79

Lebrun, F., Leray, J. P., Lavocat, P., et al. 2003, A\&A, 411, L141

Lund, N., Budtz-Jorgensen, C., Westergaaed, N. J., et al. 2003, A\&A, 411, L231
Martí, J., Mirabel, I. F., Chaty, S., \& Rodríguez, L. F. 1998, A\&A, 330,72

McLaughlin, M. A., Mattox, J. R., Cordes, J. M., \& Thompson, D. J. 1996, ApJ, 473, 763

Mukherjee, R., Halpern, J., Mirabal, N., \& Gotthelf, E. V. 2002, ApJ, 574,693

Nolan, P. L., Tompkins, W. F., Grenier, I. A., \& Michelson, P. F. 2003, ApJ, 597, 615

Paizis, A., Beckmann, V., Courvoisier, T. J.-L., et al. 2003, A\&A, 411, L363

Paul, J., Ballet, J., Cantin, M., et al. 1991, Adv. Sp. Res., 11, 289

Pavlinsky, M. N., Grebenev, S. A., \& Sunyaev, R. A. 1994, ApJ, 425, 110

Revnivtsev, M., Sunyaev, R. A., Varshalovich, D., et al. 2004, Astron. Lett., in press [arXiv:astro-ph/0402027]

Romero, G. E., Benaglia, P., \& Torres, D. F. 1999, A\&A, 348, 868

Sakano, M., Koyama, K., Murakami, H., et al. 2002, ApJS, 138, 19

Sambruna, R. M., Maraschi, L., \& Urry, C. M. 1996, ApJ, 463, 444

Sazonov, S. Yu., Revnivtsev, M. G., Lutovinov, A. A., Sunyaev, R., \& Grebenev, S. A. 2004, A\&A, 421, L21

Schlegel, D. J., Finkbeiner, D. P., \& Davis, M. 1998, ApJ, 500, 525

Sunyaev, R. A. (on behalf of the GRANAT team) 1990, IAU Circ., 5123

Sunyaev, R. A., Babichenko, S. I., Goganov, D. A., Tabaldyev, S. R., \& Iamburenko, N. S. 1990, Adv. Space Res., 10, 233

Torres, D. F., Romero, G. E., Combi, J. A., et al. 2001, A\&A, 370, 468

Ubertini, P., Lebrun, F., Di Cocco, G., et al. 2003, A\&A, 411, L131

Vedrenne, G., Roques, J. P., Schönfelder, V., et al. 2003, A\&A, 411, L63

Wallace, P. M., Halpern, J. P., Magalhaes, A. M., \& Thompson, D. J. 2002, ApJ, 569, 36

Winkler, C., Courvoisier, T. J.-L., Di Cocco, G., et al. 2003, A\&A, 411, L1 